\title{
El rol de las Facultades en la Consolidación del Estado de Derecho desde la Perspectiva del Observatorio de la Mujer
}

\author{
Laura Cristina Sánchez Frutos \\ sanchezfrutoslaura@gmail.com
}

Diana Alicia Martínez

di.aliciamartinez@gmail.com

Docentes de la Universidad Nacional de Pilar

\section{RESUMEN}

A partir de la entrada en vigencia de la Carta Magna de 1992, el Paraguay se constituye en Estado social de derecho, adoptando para su gobierno la democracia representativa, fundada en el reconocimiento de la dignidad humana. Las universidades tienen como fines formar profesionales, fomentar la investigación científica y la extensión universitaria. En este contexto, se crearon en las facultades de Derecho de las Universidades Públicas del Sur; Universidad Nacional de Itapúa y Universidad Nacional de Pilar, los Observatorios de los Derechos de la Mujer (ODM) como un espacio de análisis científico de la realidad de las mujeres en los Departamentos de Itapúa y Neembucú con la finalidad de contribuir al establecimiento de una comunidad más democrática donde puedan desarrollar su personalidad en libertad y seguridad, con pleno respeto a sus derechos. El objetivo de esta investigación es visualizar el rol de las facultades de Derecho de la UNI y la UNP en la consolidación del Estado de Derecho desde la perspectiva del Observatorio de la Mujer. El tipo de investigación correspondió a un corte transversal, el método, un enfoque cualitativo, de nivel descriptivo, donde se utilizaron técnicas tales como análisis documental, entrevista con informantes claves y cuestionario.

Palabras Claves: Estado de Derecho; Investigación; Extensión Universitaria. 


\title{
The role of the Faculties ij the consolidation of the rule of Law from the perspective of the Observatory for women
}

\begin{abstract}
As of the entry intro forcé of the 1992 Carta Magna, Paraguay has become a social state of law, adopting representative democracy for its government, based on the recognition of human dignity. Universities have the purpose of training professionals, promoting scientific research and university extension.

In this context, the wome's Rights Observatories (ODM) were created in the Faculties of Law of the Southern Public Universities, The National University of Itapúa and the National University of Pilar as a space for scientific analysis of the reality of the women in the Departaments of Itapúa and Neembucú in order to the establishment of a more democratic community where they can develop their personality in freedom and safety, with full respect for their rights. The objective of this research is of visualize the role of the UNI and UNP law schools in the consolidation of the rule of law from the perspective of the wome's Observatory. The type of research corresponded to a cross section the method, a qualitative approach, descriptive level, where techniques such as documentary analysis, interview with key informants and questionnaire.
\end{abstract}

Keywords: Rule of Law Research, University extension

Artículo recibido: 19 enero 2020

Aceptado para publicación: 22 febrero

Correspondencia: sanchezfrutoslaura@gmail.com

Conflictos de Interés: Ninguna que declarar 


\section{INTRODUCCIÓN}

El Estado de Derecho "se encuentra en la naturaleza del individuo: en la libertad y la igualdad de las personas que se reúnen en una comunidad y que se desarrollan en la autonomía moral, la igualdad jurídica y la posibilidad de lograr el bienestar”. (Marshall Barberán, 2010).

Se reconoce el proceso de construcción de la igualdad entre mujeres y hombres en el Paraguay, desde la caída de la dictadura y con años de políticas públicas plasmadas en planes, proyectos y leyes. El escenario y la coyuntura actual tienen muchos avances, pero también desafíos, que exige aspirar a más, hasta lograr la igualdad sustantiva.

En las mujeres todavía se ven vulnerados sus derechos a pesar de que los mismos están consagrados en la Carta Magna. Por tanto, los Observatorios tienen como objetivo visibilizar a las mujeres y sus derechos, para erradicar toda forma de discriminación y avanzar hacia la igualdad real y efectiva.

Las universidades públicas en Paraguay han considerado la perspectiva de género como un tema transversal en sus funciones de docencia, investigación y extensión universitaria. En este contexto, surge la idea del Observatorio de la Mujer de las universidades públicas, como una forma de contribuir al fortalecimiento del Estado de Derecho.

En el trabajo se realiza un estudio descriptivo sobre las acciones que llevan adelante los Observatorios de la Mujer de las Facultades de Derecho de las Universidades Públicas de la región sur del país, atendiendo el objetivo propuesto: el papel que cumplen los ODM en la consolidación del Estado de Derecho.

\section{Situación de la mujer en Paraguay}

Este sistema se ha legitimado a lo largo de la historia como un sistema natural o divino, señala Gena Lerner que "la subordinación de las mujeres se ve como natural y, por tanto, se torna invisible. Esto es lo que finalmente consolida con fuerza al patriarcado como una realidad y como una ideología" A su vez la Declaración sobre la Eliminación de la Violencia contra la Mujer de Naciones Unidas afirma que la violencia contra la mujer "es uno de los mecanismos sociales fundamentales por los que se fuerza a la mujer a una situación de subordinación respecto del hombre" y ésta ha permanecido oculta, silenciada y naturalizada por las propias víctimas, por las familias, bajo la tolerancia de la sociedad y los Estados, lo que la perpetua hasta ahora (Zub Centeno, 2016, pág. 14)

En el ámbito interamericano, la Convención de Belém do Pará declara en su preámbulo que "la violencia contra la mujer constituye una violación de los derechos humanos y las libertades 
fundamentales y limita total o parcialmente a la mujer el reconocimiento, goce y ejercicio de tales derechos y libertades" (Zub Centeno, 2016, pág. 18)

"En Paraguay, las mujeres de la etnia guaraní expresaron su preocupación sobre diversas formas de discriminación y violencia que sufren las comunidades a causa de los atropellos a sus territorios, a la contaminación del medio ambiente, a la falta de acceso a la salud y a una buena alimentación sobre todo cuando existen la explotación y la poca oportunidad laboral, explotación sexual y trata de personas u órganos, reafirmando que como seres humanos no se venden" (Manjoo, 2014, pág. 7)

\section{Paraguay y las leyes frente a la violencia contra de la mujer}

El Paraguay ha asumido diversos compromisos internacionales que lo obligan a eliminar la violencia y la discriminación hacia las mujeres y en esa línea se dictaron hace tiempo y más recientemente, diversas leyes que garantizan derechos específicos y sancionan su incumplimiento. También lo hacen basando su tarea en los tratados internacionales. Así, la Convención sobre la eliminación de todas formas de discriminación contra la mujer conocida como CEDAW por sus siglas en inglés, y su Protocolo Facultativo (Leyes $\mathrm{N}^{\circ} 1215 / 86^{1}$ y 1683/01²) La convención Interamericana conocida como Convención de Belém Do Pará (Ley N ${ }^{\circ}$ $\left.605 / 95^{3}\right)$.

En el ámbito interno, mencionan las normas reguladas en el Código Penal y sus leyes modificatorias, la ya antigua Ley $N^{\circ} 1.600$ “de Violencia Doméstica”, la Ley $N^{\circ}$ 5.446/15 de Políticas Públicas para las Mujeres Rurales y la nueva Ley $N^{\circ} 5.777 / 16{ }^{4}$ de Protección Integral a las Mujeres contra toda forma de violencia, cuya entrada en vigencia se produjo en los últimos días del año 2017, que implican un desafío enorme para las instituciones judiciales de todo el país y amerita un especial estudio a fin de verificar su impacto en la vigencia real de los derechos que promueven y el efectivo acceso a la justicia de las mujeres del Departamento de Neembucú, sede de la casa central de la Facultad de Derecho y Ciencias Políticas y Sociales de la UNP.

Este cúmulo de normas legales, brinda un marco que algunas veces resulta confuso, incluso para los propios actores judiciales, instituye diversas instancias donde podrían recurrir las víctimas y por ende genera diversos espacios de atención a la mujer víctima, espacios que a su vez producen

\footnotetext{
${ }^{1}$ https://www.pj.gov.py/images/contenido/secretariadegenero/marcolegal/LEY-1215-1986.pdf

${ }^{2} \mathrm{https} / / /$ www.pj.gov.py/images/contenido/secretariadegenero/marcolegal/LEY-1683.pdf

${ }^{3}$ https://www.pj.gov.py/contenido/136-secretaria-de-

genero/865\#: :text=La\%20Convenci\%C3\%B3n\%20Interamericana\%20para\%20Prevenir,y\%20goce\%20de\%20ellos\%2C\%20reconociendo

${ }^{4} \mathrm{https}: / /$ www.bacn.gov.py/leyes-paraguayas/8356/ley-n-5777-de-proteccion-integral-a-las-mujeres-contra-toda-forma-de-violencia
} 
diversas informaciones que al menos en el Departamento de Neembucú no se halla sistematizada ni reunida en un único sitio.

Por ese mismo motivo, la Ley $\mathrm{N}^{\circ}$ 5777/16 establece la creación de un Observatorio Nacional que deberá vincularse con observatorios locales que deberán actuar en red a fin de generar información interinstitucional, ya sea estadística o analítica, ya que la falta de información fidedigna se evidencia como una dificultad para el abordaje de los derechos de las mujeres, especialmente del derecho a una vida sin violencia, tal como lo reconoce el Plan Nacional contra la Violencia hacia las Mujeres 2015-20205 del Ministerio de la Mujer de Paraguay, que refiere en su fundamentación: "Los estudios que revelan las características, magnitud y evolución de la violencia contra las mujeres son realizados de manera poco sistemática en el país".

\section{METODOLOGÍA}

Se trata de una investigación cualitativa, de nivel descriptivo, con un enfoque sistémico que posibilita visualizar el fenómeno dentro de la complejidad en la que se integra y delimita su rol dentro del subsistema jurídico y del sistema social del que forma parte (Villabella Armengol, 2009,). Para el cumplimiento del objetivo planteado por el estudio, se toma como base de datos, de manera dirigida-intencional, los ODM (Observatorio de los Derechos de la Mujer), de las Facultades de Derecho de las Universidades Públicas del Sur: UNI y UNP. Las técnicas utilizadas para la recolección de datos son la revisión y análisis documental, entrevista semiestructurada con informantes claves. El tipo de investigación correspondió al corte transversal porque "recolecta los datos en un solo momento, en un tiempo único". (Hernández Sampieri, 2014)

\section{RESULTADOS Y DISCUSIÓN}

Tabla 1. Observatorio de los Derechos de la Mujer.

\begin{tabular}{|l|l|l|}
\hline El Observatorio de género de la UNI & \multicolumn{1}{|c|}{$\begin{array}{c}\text { Observatorio de los Derechos de la } \\
\text { Mujer UNP }\end{array}$} \\
\hline $\begin{array}{l}\text { El Observatorio de género de la UNI, es un } \\
\text { instrumento mediante el cual se observa, } \\
\text { analiza, procesa y teoriza acerca de la } \\
\text { realidad de las situaciones de violencia } \\
\text { contra mujeres, hombres, niños/as y y } \\
\text { adolescentes que se producen en el }\end{array}$ & $\begin{array}{l}\text { En la UNP, el Observatorio de Derechos de } \\
\text { las Mujeres, pretende constituirse en un } \\
\text { espacio de análisis científico de la realidad } \\
\text { de mujeres en el Departamento de } \\
\text { Nepartamento de Itapúa, así como las } \\
\text { consecuencias de las políticas institucionales } \\
\text { puestas en marcha, con el fin de adecuar los } \\
\text { recursos disponalidad de contribuir al } \\
\text { reales. a las necesidades }\end{array}$ & $\begin{array}{l}\text { establecimiento de una comunidad más } \\
\text { democrática donde puedan desarrollar su } \\
\text { personalidad en libertad y con seguridad, } \\
\text { con pleno respeto a sus derechos. }\end{array}$ \\
\hline
\end{tabular}

${ }^{5}$ https://docplayer.es/61185978-Ii-plan-nacional-contra-la-violencia-hacia-las-mujeres.html 


\begin{tabular}{|c|c|c|}
\hline 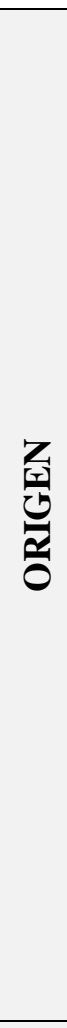 & $\begin{array}{l}\text { El ODM funciona desde el año } 2014 \text { y la } \\
\text { idea surgió gracias a una propuesta de la } \\
\text { Facultad de Ciencias Políticas de la } \\
\text { Universidad Nacional de Itapúa y el Rotary } \\
\text { Club Encarnación Norte, del que formaba } \\
\text { parte la Abg. Delssy Santa Cruz, así como la } \\
\text { psicóloga Charito Vannoli, la jueza Abg. } \\
\text { Alicia Talliercio y los señores Herman } \\
\text { Hasted y el Abg. Ricardo Ante. } \\
\text { Ahora bien, la puesta en funcionamiento y } \\
\text { recolección de datos, fue una tarea } \\
\text { exclusivamente desarrollada por la MSc. } \\
\text { María Casilda Alvarenga y la Abg. Delssy } \\
\text { Santa Cruz. El ODM está a cargo del } \\
\text { Departamento de Extensión e Investigación } \\
\text { de la Facultad de Ciencias Jurídicas de la } \\
\text { Universidad Nacional de Itapúa. }\end{array}$ & $\begin{array}{l}\text { La creación de un Observatorio de } \\
\text { Derechos de las Mujeres se halla en } \\
\text { concordancia con las líneas de } \\
\text { investigación propuestas por la Dirección } \\
\text { de Investigaciones de la Facultad de } \\
\text { Derecho, Ciencias Políticas y Sociales de } \\
\text { la UNP, aprobada por Resolución } \\
\mathrm{N}^{\circ} 370 / 2017 \text {, puesto que especialmente a } \\
\text { partir del banco de datos que se propone } \\
\text { crear, generará información pertinente para } \\
\text { analizar los problemas que se generan en la } \\
\text { aplicación real de las normas que amparan } \\
\text { los derechos de las mujeres en el territorio } \\
\text { de Ñeembucú. Para la concreción de este } \\
\text { objetivo es importante considerar que } \\
\text { actores y actoras claves en la atención de } \\
\text { mujeres víctimas de violencia en distintos } \\
\text { organismos estatales como juezas de paz y } \\
\text { juezas de garantías, fiscales, fiscalas y } \\
\text { defensoras públicas, forman parte de la } \\
\text { Facultad de Derecho, Ciencias Políticas y } \\
\text { Sociales lo que sin lugar a dudas facilita el } \\
\text { diálogo y la colaboración. }\end{array}$ \\
\hline$\frac{\mathrm{Z}}{\mathrm{O}}$ & $\begin{array}{l}\text { Brindar un espacio de información sobre } \\
\text { índices confiables de situaciones de } \\
\text { violencia contra mujeres, hombres, niños/as } \\
\text { y adolescentes que se producen en el } \\
\text { Departamento de Itapúa, como un } \\
\text { instrumento de monitoreo y transformación } \\
\text { con el fin de ejecutar iniciativas y sustentar } \\
\text { políticas de inequidad de la situación género. }\end{array}$ & \\
\hline
\end{tabular}




\begin{tabular}{|c|c|c|}
\hline$\sum_{0}^{\infty}$ & $\begin{array}{l}\text { Crear un Sistema de Información que se } \\
\text { constituya en un instrumento mediante el } \\
\text { cual se observa, analiza, procesa y teoriza la } \\
\text { realidad de situaciones de violencia contra } \\
\text { mujeres, hombres, niños/as y adolescentes } \\
\text { que se producen en el Departamento de } \\
\text { Itapúa. } \\
\text { Recabar y procesar datos de violencia contra } \\
\text { mujeres, hombres, niños/as y adolescentes } \\
\text { que se denuncian en los Juzgados de Paz, } \\
\text { Comisarias, CODENI y otros centros en el } \\
\text { Departamento de Itapúa. } \\
\text { Analizar la información recogida a través de } \\
\text { los distintos sistemas existentes. } \\
\text { Elaborar indicadores de seguimiento y } \\
\text { evaluación de la situación. } \\
\text { Diseñar y poner en funcionamiento una } \\
\text { aplicación informática que almacene y } \\
\text { procese los datos relativos a las situaciones } \\
\text { de violencia de género. } \\
\text { Divulgar información sistemática y veraz de } \\
\text { datos cuantitativos a la comunidad en } \\
\text { general. } \\
\text { Sensibilizar a la población sobre la violencia } \\
\text { doméstica promoviendo una cultura de } \\
\text { igualdad, diálogo, respeto, práctica de } \\
\text { valores y del buen trato. }\end{array}$ & $\begin{array}{l}\text { El ODM se propone, inicialmente } \\
\text { desarrollar sus acciones en relación a dos } \\
\text { temas específicos: Violencia de género y } \\
\text { Discriminación hacia mujeres, en } \\
\text { Neembucú. }\end{array}$ \\
\hline
\end{tabular}




\begin{tabular}{|c|c|c|}
\hline 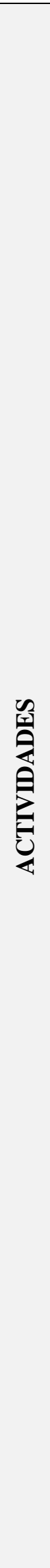 & $\begin{array}{l}\text { Se circunscriben a la recopilación, } \\
\text { incorporación y actualización de los } \\
\text { indicadores del observatorio. Estas son: } \\
\text { Construcción de las variables y sus } \\
\text { definiciones para cada indicador. } \\
\text { Elaboración de las fichas técnicas. } \\
\text { Metodología de procesamiento y medida } \\
\text { sobre las fuentes y las dimensiones; } \\
\text { desagregaciones específicas de cada } \\
\text { denuncia. Recopilación, actualización y } \\
\text { análisis de la información. } \\
\text { Talleres para recolectores de información. }\end{array}$ & $\begin{array}{l}\text { Recopilar y sistematizar noticias y } \\
\text { estadísticas sobre casos de violencia o } \\
\text { discriminación hacia mujeres y niñas en el } \\
\text { Departamento de Neembucú de fuentes } \\
\text { primarias (instituciones estatales) y } \\
\text { secundarias (medios de comunicación, } \\
\text { entrevistas personales) a fin de ofrecer } \\
\text { insumos para el establecimiento de } \\
\text { políticas públicas } \\
\text { 1.1 Crear y administrar un sitio web donde } \\
\text { se difundan esas noticias y estadísticas. } \\
\text { 1.2 Celebrar convenios y acuerdos con } \\
\text { instituciones del área a fin de intercambiar } \\
\text { información y estadísticas. } \\
\text { 2. Vigilar e incidir en los procesos de } \\
\text { formulación e implementación de leyes, } \\
\text { normas, protocolos y sentencias sobre } \\
\text { violencia de género y discriminación hacia } \\
\text { las mujeres y niñas con un enfoque } \\
\text { científico y propositivo. } \\
\text { 3. Evaluar y dar seguimiento a las acciones, } \\
\text { omisiones, avances o retrocesos en favor } \\
\text { de los derechos de las mujeres y la } \\
\text { eliminación de la violencia hacia ellas en el } \\
\text { ámbito nacional y especialmente } \\
\text { departamental. } \\
\text { 4. Propiciar investigaciones científicas } \\
\text { sobre la vigencia de los derechos de las } \\
\text { niñas y mujeres en Neembucú y } \\
\text { especialmente sobre violencia hacia las } \\
\text { mujeres y niñas. } \\
\text { 4.1. Propiciar un programa de incentivos } \\
\text { para docentes que se dediquen a la } \\
\text { investigación es el área de interés del } \\
\text { ODM, ante la UNP u otros organismos. } \\
\text { 4.2. Propiciar la creación de un fondo } \\
\text { concursable para financiar esas } \\
\text { investigaciones en el ámbito universitario. } \\
\text { 5. Monitorear las buenas prácticas y las } \\
\text { dificultades generadas en la aplicación de } \\
\text { leyes a favor de los derechos de las niñas y } \\
\text { mujeres y especialmente de las normas } \\
\text { sancionadas recientemente en el país y en } \\
\text { el ámbito departamental. } \\
\text { 6. Generar espacios de diálogo y reflexión } \\
\text { crítica sobre las relaciones sociales, } \\
\text { culturales económicas y jurídicas en que se } \\
\text { desarrollan en Ñeembucú desde una } \\
\text { perspectiva de género y en especial } \\
\text { relación a la violencia y discriminación } \\
\text { hacia mujeres y niñas. } \\
\text { 7. Vincular a la Facultad de Derecho, } \\
\text { Ciencias Políticas y Sociales de la UNP }\end{array}$ \\
\hline
\end{tabular}




\begin{tabular}{|l|l|l|}
\hline & $\begin{array}{l}\text { con organismos nacionales, } \\
\text { internacionales, públicos y privados } \\
\text { interesados en esta problemática, a fin de } \\
\text { participar en redes, programas de } \\
\text { investigación conjunta, campañas de } \\
\text { difusión u otras actividades que faciliten la } \\
\text { concreción de los objetivos de este } \\
\text { proyecto. }\end{array}$ \\
\hline
\end{tabular}

Elaboración propia.

\section{Resultados recabados de informantes claves.}

A través de la justificación académica se procedió a la constitución de un grupo de investigadoras, compuesta por la Abg. Marialba Campias, Lic. María Dolores Muñoz, Lic. Elida Duarte, y Lic. Romina Ríos. Esto permitió la realización de un trabajo multidisciplinario con profesionales del Derecho y Trabajo Social en este Observatorio, para analizar una problemática vigente en la comunidad, desde la perspectiva de varias materias de la carrera de Derecho, pues se aplican contenidos que se desarrollan en cátedras tales como Derecho Constitucional, Derechos Humanos, Criminología, Derecho Penal, Derecho Civil (Personas y Familia).

La creación de un Observatorio de Derechos de las Mujeres se halla en concordancia con las líneas de investigación propuestas por la Dirección de Investigaciones de la Facultad de Derecho, Ciencias Políticas y Sociales de la UNP, aprobada por Resolución N`370/2017, puesto que especialmente a partir del banco de datos que se propone crear, genera información pertinente para analizar los problemas que aparecen en la aplicación real de las normas que amparan los derechos de las mujeres, en el territorio de Neembucú. Para la concreción de este objetivo es importante considerar que actores y actoras claves en la atención de mujeres víctimas de violencia en distintos organismos estatales como juezas de paz y juezas de garantías, fiscales, fiscalas y defensoras públicas, forman parte también de la Facultad lo que, sin lugar a dudas facilitará, el diálogo y la colaboración.

Además, el ODM puede contribuir a generar un lazo con la comunidad mediante la investigación y la extensión universitaria, vinculando a la facultad con otras instituciones y también puede ser un espacio para la práctica de los y las estudiantes en esas misiones fundamentales de los estudios superiores. Proyectos similares al que aquí se propone, se vienen desarrollando en otros países (Colombia, Méjico, Venezuela, por ejemplo) y en muchos casos son justamente las universidades, en su rol de investigación y extensión, quienes suelen organizarlos en algunos casos con asistencia de organismos internacionales como ONU MUJERES. 


\section{CONCLUSIÓN O CONSIDERACIONES FINALES}

Los ODM de las universidades de la región sur del país están cumpliendo un papel sumamente interesante, al plantearse trabajar en la promoción de los derechos de las mujeres, consideradas como población vulnerable dentro de un sistema patriarcal. Se constituyen en el nexo entre la academia y la sociedad, a través de las diferentes actividades planteadas, mediante la extensión universitaria y la investigación científica, consideradas funciones inherentes a las instituciones de Educación Superior, consiguiendo la transferencia del conocimiento.

Los ODM se proponen objetivos similares que encaran de maneras diferentes, conforme a las características propias de la población a la que se dirigen, así como los profesionales comprometidos con esta labor. Sistematización de los datos obtenidos, para los lineamientos de las políticas públicas de los derechos de las mujeres. Favorecer las buenas prácticas y las dificultades generadas en la aplicación de leyes en favor de los derechos de las niñas y mujeres, involucrando a estudiantes del primer año de la carrera de Derecho, así como vincular a docentes y toda la comunidad educativa a través de diversas cátedras de Derecho. Concienciar y promover la reflexión crítica sobre las relaciones de poder desde una perspectiva de género.

Se considera que las facultades de Derecho analizadas están contribuyendo a la consolidación del Estado de Derecho, con numerosos logros, mediante las actividades de los ODM, a pesar de las dificultades que han señalado.

A través de la investigación realizada se unifican criterios en pos a los derechos de las mujeres, es necesario avanzar hacia otros aspectos de relevancia para incrementar el impacto en las poblaciones a las que van dirigidas e incidir en las políticas públicas integrales.

\section{REFERENCIAS BIBLIOGRAFICAS}

Hernández Sampieri, R. (2014). Definición del alcance de la investigación que se realizará: exploratorio,descriptivo, correlacional o explicativo. E -uaem - Espacio de Formación Multimodal, págs. 88-101.

Manjoo, R. (2014). Patrones de Violencia contra las Mujeres en América Latina y el Caribe. Informe presentado a la Relatora de la ONU sobre Violencia contra la Mujer, sus Causas y Consecuencias. Perú: (C) Comité de América Latina y el Caribe para la Defensa de los Derechos de las Mujeres - CLADEM.

Marshall Barberán, P. (2010). EL ESTADO DE DERECHO COMO PRINCIPIO Y SU CONSAGRACIÓN EN LA CONSTITUCIÓN POLÍTICA. Revista de Derecho Universidad Católica del Norte, 185 - 204. 
Ministerio de la mujer . (2020). Observatorio de la mujer. Para los seguimientos sobre Violencia hacia las mujeres. Obtenido de http://observatorio.mujer.gov.py/index.php/institucion/marco-legal

Villabella Armengol, C. (2009,). La investigación científica en la ciencia jurídica. Sus particularidades. IUS. Revista del Instituto de Ciencias Jurídicas de Puebla A.C., núm. 23, , pp. 5-37.

Zub Centeno, M. (2016). Violencia Contra las Mujeres en Paraguay: Avances y Desafios. Asunción: ONU Mujeres. 